\title{
Entangling the free motion of a particle pair: an experimental scenario
}

\author{
Clemens Gneiting and Klaus Hornberger \\ Arnold Sommerfeld Center for Theoretical Physics, \\ Ludwig-Maximilians-Universität München, Theresienstraße 37, 80333 Munich, Germany
}

(Dated: November 24, 2018)

\begin{abstract}
The concept of dissociation-time entanglement provides a means of manifesting non-classical correlations in the motional state of two counter-propagating atoms. In this article, we discuss in detail the requirements for a specific experimental implementation, which is based on the Feshbach dissociation of a molecular Bose-Einstein condensate of fermionic lithium. A sequence of two magnetic field pulses serves to delocalize both of the dissociation products into a superposition of consecutive wave packets, which are separated by a macroscopic distance. This allows to address them separately in a switched Mach-Zehnder configuration, permitting to conduct a Bell experiment with simple position measurements. We analyze the expected form of the two-particle wave function in a concrete experimental setup that uses lasers as atom guides. Assuming viable experimental parameters the setup is shown to be capable of violating a Bell inequality.
\end{abstract}

PACS numbers: 03.67.Bg, 37.25.+k, 03.65.Ud

published in Opt. Spectroscop. 108, 188 (2010)

\section{INTRODUCTION}

The increasing control of quantum systems that has been obtained in the last decades brings into reach the possibility of performing hitherto unprecedented tests of the principles of quantum mechanics [1 - 5 ]. In particular, there is a growing interest in demonstrating nonclassical behavior in systems or with respect to features that would be assigned to the classical realm according to our every-day experience. One exciting approach aims at luring mesoscopic mechanical oscillators into the quantum regime, see [ [6] and refs therein. Another approach focuses on the generation of highly non-classical states of continuous variables with direct classical correspondence, such as creating superpositions of macroscopically different phase space coordinates $[-7-10]$. The recent achievements in the generation and manipulation of ultracold atoms and molecules [11 16] suggest to imprint non-classical features even on the free motion of material particles. For example, it has been proposed to perform the original Einstein-Podolsky-Rosen thought experiment based on the dissociation of a molecular BoseEinstein condensate (BEC) [17, 18].

In a recent article we go one step further by proposing to violate a Bell inequality in the motional state of material particles using position measurements in the end [19]. The two-particle state considered in this proposal can be characterized by its generation procedure. Starting from a diatomic Feshbach molecule, which is trapped in an optical dipole trap, a sequence of two short magnetic field pulses creates the superposition of two subsequent dissociations. Due to momentum conservation the dissociated atoms propagate in opposite directions along some wave guide, with each atom delocalized into a pair of macroscopically separated, consecutive wave packets, corresponding to the early and the late dissociation instant, see Fig. 1. An observer cannot tell whether the dissociation took place at the early or at the late instant, while both atoms must have started their journey at the same time. In this sense, the generated two-particle state may be called dissociation-time entangled (DTE).

Since each atom in a DTE state is described by two spatially distinct propagating wave packets, which correspond to the early and the late dissociation times, the use of switched, asymmetric Mach-Zehnder interferometers can serve first to endow these wave packets with additional relative phases and then to rejoin them. In detail, the early wave packets propagate through the long arms of the interferometers, whereas the late ones are directed through the short arms, see Fig. 1 c). If the detour of the early wave packets is chosen appropriately, they rejoin the late ones in the final beam splitters. Provided the two dissociation processes are coherent in the sense that no information about the dissociation time has leaked to the environment, the rejoined wave packets may interfere. The joint probability for detecting the particles in the output ports of the two interferometers then exhibits a fringe pattern under variation of either path length difference, which is in principle capable of violating a Bell inequality. We emphasize that the only relevant information required to extract these correlations is the output port combination where the atoms are detected. This information can be obtained by simple position measurements, without the need of a particular spatial or temporal resolution. And since the position measurements can be understood from a classical physicist's point of view, they are indeed in line with the above mentioned pursuit of focusing on features that have a classical analogoue.

We note that a similar Bell test procedure was first introduced for photons in 20, 21]. This led to the experimental demonstration of a Bell inequality violation for parties separated by several kilometers, albeit using a postselection procedure required due to the lack of sufficiently good switches for photons. The implications for material particles, in particular the detrimental effect of dispersion, are discussed in detail in [22].

In this article we devise a concrete scenario to perform the described DTE Bell test, which is sufficiently detailed 
a)

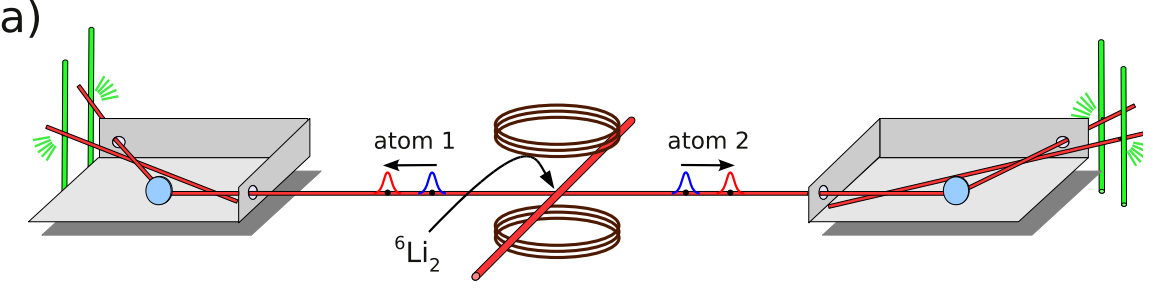

b)

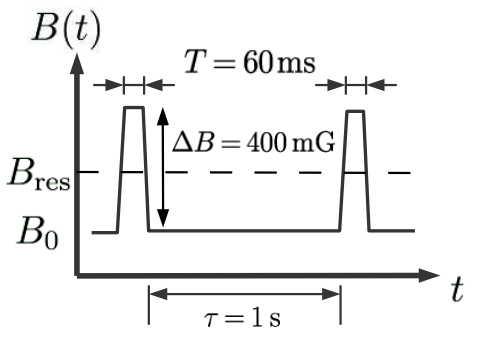

c)

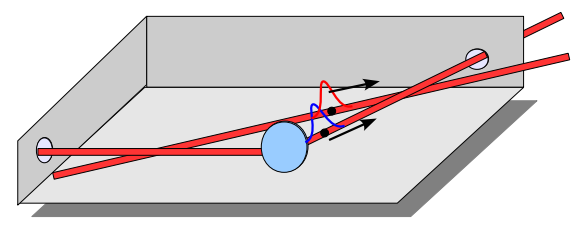

FIG. 1: (color online) a) Experimental setup for the DTE Bell test. It starts with a ${ }^{6} \mathrm{Li}_{2}$ Bose-Einstein condensate of about $10^{2}$ molecules in an optical dipole trap. A sequence of two short magnetic field pulses, as shown in b), dissociates on average one molecule per shot into a pair of counter-propagating atoms. The resulting two-particle state corresponds to a superposition of two subsequent dissociations, where each atom is delocalized into two consecutive wave packets that are separated by a distance of $5 \mathrm{~mm}$. The two atoms propagate in opposite directions along the laser guide and eventually enter switched, asymmetric MachZehnder interferometers that are implemented by two more laser beams crossing the guide in a triangular arrangement at small angles. These second lasers are switched on after the early wave packets have passed, but before the late wave packets arrive. An additional perpendicular, blue-detuned blocking laser beam, which is not depicted in the figure, may be used to support the deflection of the late wave packets. This way, the early wave packets have to take the long paths, whereas the late ones are directed into the short paths, as depicted in c). If the path length differences cancel the distance between the early and the late wave packets, they interfere and the joint probability of detection in the output ports of the interferometers exhibits a fringe pattern as a function of the path length difference variation. Correlations of the single-particle detection events thus have the potential to violate a Bell inequality.

and realistic to suggest that violating a Bell inequality should be possible within reach of present day technology. Section II provides a detailed quantitative account of the proposed setup, based on realistic choices for the experimental parameters. It involves pulse separations on the order of seconds and implies atom velocities on the order of centimeters per second, as well as a truly macroscopic spatial separation of the single atom wave packets on the order of centimeters. In Section III we discuss and numerically evaluate the two-particle superposition state that is generated in the wave guide in the course of a double dissociation with two square pulses. Section IV demonstrates that this state, if exposed to the switched, asymmetric Mach-Zehnder processing, generates a fringe pattern which violates a Bell inequality. Finally, we conclude in Section $\nabla$ by pointing out the advantages of the proposed scheme.

\section{EXPERIMENTAL SCENARIO FOR A DTE BELL TEST}

In the following we discuss quantitative details of a possible experimental setup that should be capable of generating a pair of dissociation-time entangled atoms and to violate a Bell inequality in terms of single-particle interferometry and simple position measurements. We work at this example to prove the viability of the proposal presented in [19], but of course many alternative implementations are conceivable. We emphasize that the setup is designed to allow for time and length scales on the order of seconds and centimeters, establishing entanglement with respect to single-particle properties that are truly macroscopically distinct. In this section we focus on a consistent set of experimental parameters, deferring the theoretical analysis of the parts concerning the dissociation process to Section [II]

We suggest to use a dilute molecular Bose-Einstein condensate (BEC) produced from a 50:50 spin mixture of fermionic ${ }^{6} \mathrm{Li}$ as a starting point. Such a fermionic mixture seems favorable compared to bosonic ingredients, since huge lifetimes of more than 10s can be achieved due to Pauli blocking of detrimental 3-body collisions [12]. This makes truly macroscopic time separations $\tau$ between the two dissociation pulses conceivable, and we choose $\tau=1 \mathrm{~s}$ in the following. It has been demonstrated that the molecular ${ }^{6} \mathrm{Li} \mathrm{BEC}$ can be prepared efficiently and with near-perfect purity [12], and the comparatively small mass of lithium reconciles reasonable propagation velocities, on the order of $1 \mathrm{~cm} / \mathrm{s}$, with resolvable de Broglie wave lengths, on the order of $10 \mu \mathrm{m}$.

The BEC is prepared in an optical dipole trap created 
by two red-detuned, far off-resonant, crossing laser beams with a wave length of, say $\lambda=1 \mu \mathrm{m}$, see Fig. 1 a). The guiding laser, which connects the two single-atom interferometers, serves as an atom guide which compensates the gravitational force. Moreover, it must be chosen sufficiently strong that the transverse motion of the atoms remains frozen in the ground state. This way an effectively one-dimensional description of the atomic motion can be used.

The following numerical values are chosen to be consistent with a pulse separation of $\tau=1 \mathrm{~s}$ and a relative velocity of $v_{\text {rel }}=1 \mathrm{~cm} / \mathrm{s}$. We assume that the guiding laser is given by a Gaussian laser beam and operated at a power of $P_{\mathrm{G}}=32.85 \mathrm{~W}$ with a (center) waist of $w_{0, \mathrm{G}}=216 \mu \mathrm{m}$, such that the optical dipole potential resulting from a molecular polarizability of $\alpha_{\mathrm{Li}_{2}}(\lambda=$ $1 \mu \mathrm{m})=88.5 \AA^{3}$ has a (center) trap depth of $30 \mu \mathrm{K}$. It thus is strong enough to compensate gravitation, supporting several thousand transversally bound states. The implied Rayleigh length of about $z_{0, \mathrm{G}}=15 \mathrm{~cm}$ sets the scale for the extension of the whole setup. Decoherence due to photon scattering can be estimated to occur at a rate on the order of $0.05 \mathrm{~s}^{-1}$, which limits the overall duration of a single experiment to remain below $20 \mathrm{~s}$. The implied transverse trap frequency of $\omega_{\mathrm{G}} / 2 \pi=300 \mathrm{~Hz}$, on the other hand, limits the maximum dissociation velocity not to exceed about $v_{\text {rel }}=1 \mathrm{~cm} / \mathrm{s}$, if we demand the transverse motion of the atoms to remain frozen in the ground state. The corresponding de Broglie wave length $\lambda_{\text {rel }}=12.4 \mu \mathrm{m}$, which sets the scale for the fringe separation, is in compliance with viable stability requirements for the interferometers.

The second laser beam intersects the guiding laser perpendicularly, creating an elongated dipole trap for the $\mathrm{BEC}$ within the laser guide. At the same time, it must be sufficiently shallow to be overcome with reasonable magnetic field pulses. Moreover, the resulting longitudinal trap ground state $\left|\psi_{\mathrm{T}}\right\rangle$, which is supposed to determine the center of mass state of the trapped molecules, must meet stringent conditions regarding its momentum spectrum. Only a sufficiently narrow momentum spectrum guarantees that the dispersion-induced reduction of the non-local correlations due to the momentum spread in the center of mass motion admits the violation of a Bell inequality [19]. This demands a comparatively small trap frequency. Specifically, for an atomic propagation velocity of $5 \mathrm{~mm} / \mathrm{s}$, the trap frequency should not greatly exceed $\omega_{\mathrm{T}} / 2 \pi=0.25 \mathrm{~Hz}$.

A reasonable trap depth of $U_{\mathrm{T}} / k_{\mathrm{B}}=50 \mathrm{nK}$ combined with the required trap frequency of $\omega_{\mathrm{T}} / 2 \pi=0.25 \mathrm{~Hz} \mathrm{im}$ plies the trap laser (center) beam waist to be $w_{0, \mathrm{~T}}=$ $1.1 \mathrm{~cm}$. By employing an elliptic Gaussian laser beam with waist ratio $w_{x, \mathrm{~T}} / w_{y, \mathrm{~T}}=10$, the required laser power can be kept reasonably low, at $P_{\mathrm{T}}=13.1 \mathrm{~W}$. The corresponding photon scattering rate of $10^{-4} \mathrm{~S}^{-1}$, on the other hand, does not impose any limitations.

The preparation is arranged such that only a small number of molecules, on the order of $10^{2}$, remains in the BEC at the end. These can be taken to be noninteracting, so that the initial longitudinal center of mass state of the molecules indeed is given by the trap laser ground state, parametrized by the trap frequency $\omega_{\mathrm{T}}$.

A DTE atom pair is extracted from the BEC by applying a sequence of two short square pulses in the external magnetic field, as shown in Fig. 1 b). The pulses must be chosen such that the desired dissociation velocity of $v_{\text {rel }}=1 \mathrm{~cm} / \mathrm{s}$ is accomplished. To this end the pulses not only have to provide the corresponding kinetic energy but also the energy to overcome the longitudinal trap depth of $U_{\mathrm{T}}=k_{\mathrm{B}} 50 \mathrm{nK}$. Moreover, the generated state of the relative motion must meet similarly stringent conditions concerning its momentum spectrum as the trap laser ground state $\left|\psi_{\mathrm{T}}\right\rangle$.

The desired dissociation velocity of $v_{\text {rel }}=1 \mathrm{~cm} / \mathrm{s}$ is obtained for a narrow, sufficiently isolated resonance with, e.g., resonance width $\Delta B_{\text {res }}=1 \mathrm{mG}$ and $\mu_{\text {res }}=0.01 \mu_{\mathrm{B}}$, by applying a pulse duration of $T=60 \mathrm{~ms}$ and a pulse height of $B_{0}+\Delta B-B_{\text {res }}=200 \mathrm{mG}$. Here, $\mu_{\text {res }}$ denotes the difference between the magnetic moments of the resonance state and the open channel, $B_{0}$ stands for the magnetic field base value before and after the pulses, and $B_{\text {res }}$ indicates the resonance position. In particular, the generated state meets the tight restrictions on the momentum distribution as imposed by dispersion. In Section III we will analyze the momentum spectrum resulting from such a double pulse sequence and explicitly demonstrate its capability of violating a Bell inequality.

The dissociation probability per double pulse sequence depends, apart from the shape of the magnetic field pulses, on the resonance parameters $\Delta B_{\text {res }}$ and $\mu_{\text {res }}$, on the guiding laser trapping frequency $\omega_{\mathrm{G}}$, and on the background channel scattering length $a_{\mathrm{bg}}$. For a generic value of $a_{\mathrm{bg}}=100 a_{0}$ the dissociation probability amounts to a few percent. We thus rely on post-selection in order to guarantee that only one atom pair per shot enters the interferometers. This is however unproblematic, since the final fluorescence detection of the slow, strongly confined atoms can be done with single particle resolution, so that it is easy to disregard the cases of too many atom pairs in the process. For a start, we stick to this simple post-selection procedure; in a more refined setup it is conceivable to use a specially prepared optical lattice where each site is occupied by at most one molecule [23].

After the completion of the dissociation sequence, and for our chosen time separation of $\tau=1$ s between the early and the late dissociation pulse, the corresponding early and late wave packets of each particle propagate at a velocity of $5 \mathrm{~mm} / \mathrm{s}$, separated by a distance of $5 \mathrm{~mm}$ on each side. This constitutes a truly macroscopic delocalization of each atom. Immediately after the dissociation process, the widths of the early and late single-particle wave packets are on the order of about $200 \mu \mathrm{m}$. Their narrow momentum spectra guarantee that these wave packet extensions are not appreciably modified during the propagation to the interferometers if the propagation time does not exceed about 10s. The early and late 
wave packets are thus spatially still sufficiently distinct when arriving at the interferometers for the switches to be applicable.

The interferometers are implemented by two more reddetuned laser beams crossing the guide in a triangular arrangement at small angles, see Fig. 1. The path length differences must compensate the $5 \mathrm{~mm}$ distance between the early and late wave packets of each particle. Small variations of the path length differences on the order of the de Broglie wave length $\lambda_{\text {rel }}=12.4 \mu \mathrm{m}$ result in a variation of the relative phase between the early and the late wave packets, as required for the Bell test [19]. The additional laser beams are switched on only after the early wave packets have passed the crossing points, but before the late ones arrive there. The crossings of the beams then act as beam splitters, while the required atom mirrors may be realized using evanescent light fields or bluedetuned laser beams perpendicular to the interferometer planes [24, 25]. Such perpendicular blocking beams at the crossing point of the first beam splitters could also support the full deflection of the late wave packets into the short arms. This way, the early wave packets pass through the long arms, whereas the late wave packets are deflected into the short arms, as depicted in Fig. 1] c). Note that a simplified setup could do without the switching, replacing the switches by ordinary beam splitters, at the cost of $50 \%$ post-selection.

Resonant laser beams crossing the guiding lasers behind the final beam splitters, as sketched in Fig. 1 a), effect fluorescence detection with near unit efficiency and with single particle resolution. No particular spatial or temporal resolution of the detection is required, since the only relevant information for the Bell test is the combination of beam splitter output ports where the particles are detected. Similar to the original Bell test based on spins, the joint probability distribution for detecting the particles in particular output port combinations shows an interference pattern under variation of either path length difference, which can violate a Bell inequality. Decoherence due to photon scattering in the guiding laser beam sets the scale for the distance between the BEC trap and the detection lasers to be on the order of $10 \mathrm{~cm}$ in our example.

\section{DTE STATE FROM A DOUBLE DISSOCIATION PULSE}

We proceed to derive the asymptotic form of the twoparticle state that is generated by a double pulse sequence in the described setup, and we verify that it has the DTE structure as described in Section [I. In particular, we will be able to specify the corresponding momentum spectrum, which plays a crucial role for the feasibility of the Bell test. Below in Section IV we will use this result to demonstrate explicitly that the experimental scenario presented above yields a DTE state which can violate a Bell inequality in spite of wave packet dis- persion.

A two-channel single-resonance calculation shows that after an arbitrary magnetic field pulse sequence (close to an isolated resonance) the dissociated part of the state, $\left|\Phi_{\mathrm{bg}}(t)\right\rangle$, is described, for low energies, at positions far from the dissociation center, and at large times, by the asymptotic form

$$
\left|\Phi_{\mathrm{bg}}(t)\right\rangle \sim C_{\mathrm{bg}}\left|\varphi_{0,0}^{\mathrm{cm}}\right\rangle\left|\varphi_{0,0}^{\mathrm{rel}}\right\rangle \widehat{\mathrm{U}}_{z, t}^{(0)}\left|\Psi_{z}\right\rangle .
$$

Here $\widehat{\mathrm{U}}_{z, t}^{(0)}$ denotes the free (two-particle) propagator in the longitudinal direction, while the transverse motion is frozen in the harmonic ground state, $\left|\varphi_{0,0}^{\mathrm{cm}}\right\rangle$ and $\left|\varphi_{0,0}^{\mathrm{rel}}\right\rangle$, resp., of the guiding laser beam. The longitudinal part of the two-particle state, expressed in the basis of the center of mass and relative momenta $p_{\mathrm{cm}}$ and $p_{\text {rel }}$, is determined by [19]

$$
\left\langle p_{\mathrm{cm}}, p_{\mathrm{rel}} \mid \Psi_{z}\right\rangle=\frac{\tilde{C}\left(p_{\mathrm{cm}}^{2} / 4 m \hbar+p_{\mathrm{rel}}^{2} / m \hbar+2 \omega_{\mathrm{G}}\right)}{\|\tilde{C}\|}\left\langle p_{\mathrm{cm}} \mid \psi_{\mathrm{T}}\right\rangle,
$$

where $\tilde{C}(\omega)$ is the Fourier transform of the closed channel probability amplitude $C(t), \tilde{C}(\omega)=\int_{-\infty}^{\infty} \mathrm{d} t \mathrm{e}^{\mathrm{i} \omega t} C(t)$. The quantity

$$
\begin{aligned}
\|\tilde{C}\|^{2}= & \int_{-\infty}^{\infty} \mathrm{d} p_{\mathrm{cm}} \int_{-\infty}^{\infty} \mathrm{d} p_{\mathrm{rel}}\left|\tilde{C}\left(\frac{p_{\mathrm{cm}}^{2}}{4 m \hbar}+\frac{p_{\mathrm{rel}}^{2}}{m \hbar}+2 \omega_{\mathrm{G}}\right)\right|^{2} \\
& \times\left|\left\langle p_{\mathrm{cm}} \mid \psi_{\mathrm{T}}\right\rangle\right|^{2}
\end{aligned}
$$

normalizes the spectrum. The dissociation probability can be estimated by

$$
\left|C_{\mathrm{bg}}\right|^{2}=\frac{\omega_{\mathrm{G}} a_{\mathrm{bg}} \mu_{\mathrm{res}} \Delta B_{\mathrm{res}}}{\pi \hbar^{2}}\|\tilde{C}\|^{2}
$$

which involves the background scattering length $a_{\mathrm{bg}}$, the resonance width $\Delta B_{\text {res }}$, and $\mu_{\text {res }}$, the difference between the magnetic moments of the resonance state and the open channel. As mentioned in Section II, we presume a narrow resonance width of $\Delta B_{\text {res }}=1 \mathrm{mG}$ and short pulse durations $T=60 \mathrm{~ms}$, such that only a small fraction of the condensate dissociates. The coupling between the two channels is then sufficiently weak so that the backaction from the open channel to the bare resonance state can be safely neglected. In this case, a time-dependent magnetic field $B(t)$ determines the closed channel probability amplitude according to

$$
C(t)=C\left(t_{0}\right) \exp \left(-\frac{\mathrm{i}}{\hbar} \int_{t_{0}}^{t} \mathrm{~d} t^{\prime}\left[E_{\mathrm{res}}\left(B\left(t^{\prime}\right)\right)-2 U_{\mathrm{T}}+\hbar \omega_{\mathrm{G}}\right]\right) .
$$

The resonance state energy $E_{\text {res }}(B(t))=\mu_{\text {res }}\left(B(t)-B_{\text {res }}\right)$ is measured with respect to the background channel continuum threshold, whereas the trap laser potential depth $U_{\mathrm{T}}$ and the transverse trap frequency of the guiding laser $\omega_{\mathrm{G}}$ cause an additional energy offset that has to be overcome in the dissociation process. $C\left(t_{0}\right)$ indicates the probability amplitude for the two particles to be found in 
the resonance state at some initial time $t_{0}$ well before the dissociation pulses take place. Since we can presume that the magnetic field before $t_{0}$ has remained well below the resonance $B_{\text {res }}$, the corresponding background channel component is negligible and we can safely set $C\left(t_{0}\right)=1$ from now on.

For a sequence of two square pulses, each of height $\Delta B$ and duration $T$, separated by the time $\tau$,

$$
B(t)=B_{0}+\Delta B \theta\left(t+\tau+\frac{T}{2}\right) \theta\left(-\tau+\frac{T}{2}-t\right)+\Delta B \theta\left(t+\frac{T}{2}\right) \theta\left(\frac{T}{2}-t\right)
$$

the Fourier transform of (4) can be evaluated analytically, yielding

$$
\begin{aligned}
\tilde{C}(\omega)= & \frac{T \mu_{\mathrm{res}} \Delta B \operatorname{sinc}\left[\left(\omega-\mu_{\mathrm{res}}\left[B_{0}+\Delta B-B_{\mathrm{res}}\right] / \hbar+2 U_{\mathrm{T}} / \hbar-\omega_{\mathrm{G}}\right) T / 2\right]}{\hbar \omega-\mu_{\mathrm{res}}\left[B_{0}-B_{\mathrm{res}}\right]+2 U_{\mathrm{T}}-\hbar \omega_{\mathrm{G}}} \\
& \times\left\{\mathrm{e}^{-\mathrm{i} \omega \tau}+\mathrm{e}^{\mathrm{i}\left[2 U_{\mathrm{T}} \tau-\mu_{\mathrm{res}} \Delta B T+\mu_{\mathrm{res}}\left(B_{\mathrm{res}}-B_{0}\right) \tau-\hbar \omega_{\mathrm{G}} \tau\right] / \hbar}\right\},
\end{aligned}
$$

where we have neglected an irrelevant global phase. It is now helpful to introduce the mean energy

$$
\frac{p_{0}^{2}}{m}=\mu_{\mathrm{res}}\left(B_{0}+\Delta B-B_{\mathrm{res}}\right)-2 U_{\mathrm{T}}-\hbar \omega_{\mathrm{G}},
$$

the characteristic width

$$
\Delta p^{2}=2 m \hbar / T
$$

and the pulse energy

$$
\bar{p}^{2} / m=\mu_{\text {res }} \Delta B .
$$

The dissociated state (1) thus takes the form

$$
\begin{aligned}
\|\tilde{C}\|\left\langle p_{\mathrm{cm}}, p_{\text {rel }} \mid \Psi_{z}\right\rangle= & T \bar{p}^{2}\left\langle p_{\mathrm{cm}} \mid \psi_{\mathrm{T}}\right\rangle \\
& \times \frac{\operatorname{sinc}\left[\left(p_{\mathrm{cm}}^{2} / 4+p_{\mathrm{rel}}^{2}-p_{0}^{2}\right) / \Delta p^{2}\right]}{p_{\mathrm{cm}}^{2} / 4+p_{\text {rel }}^{2}-p_{0}^{2}+\bar{p}^{2}} \\
& \times\left\{\mathrm{e}^{-\mathrm{i}\left(p_{\mathrm{cm}}^{2} / 4 m \hbar+p_{\text {rel }}^{2} / m \hbar\right) \tau}+\mathrm{e}^{\mathrm{i} \phi_{\tau}}\right\},
\end{aligned}
$$

where the relative phase between the early and the late dissociation component is given by

$$
\phi_{\tau}=\left[2 U_{\mathrm{T}} \tau-\mu_{\mathrm{res}} \Delta B T+\mu_{\mathrm{res}}\left(B_{\mathrm{res}}-B_{0}\right) \tau\right] / \hbar+\omega_{\mathrm{G}} \tau .
$$

For the choice of parameters presented in Section II the momentum spectrum (7) is sharply peaked at $\left(p_{\mathrm{cm}}, p_{\text {rel }}\right)=\left(0, \pm p_{0}\right)$, with $p_{0} / m=5 \mathrm{~mm} / \mathrm{s}$. This is demonstrated by considering the ratios $\sigma_{p, \mathrm{~T}} / p_{0}$ and $\Delta p / p_{0}$, where $\sigma_{p, \mathrm{~T}}$ denotes the momentum uncertainty exhibited by the longitudinal trap ground state $\left|\psi_{\mathrm{T}}\right\rangle$, reading in the harmonic approximation $\sigma_{p, \mathrm{~T}}=\sqrt{\hbar \omega_{\mathrm{T}} m}$. This yields the ratio $\sigma_{p, \mathrm{~T}} / p_{0}=0.024$, while the pulse duration $T=60 \mathrm{~ms}$ implies $\Delta p / p_{0}=0.11$. We can exploit the smallness of these ratios in order to calculate the normalization $\|\tilde{C}\|^{2}$ according to (2). For this, we can focus on a single-pulse contribution, since in the limit $\tau \gg T$ the spectrum (17) represents the superposition of two spatially distinct dissociation states, which contribute equally to the norm. Within the range $\sigma_{p, \mathrm{~T}}$ where $\left\langle p_{\mathrm{cm}} \mid \psi_{\mathrm{T}}\right\rangle$ is non-negligible, the Feshbach contribution $\tilde{C}\left(p_{\mathrm{cm}}^{2} / 4 m \hbar+p_{\text {rel }}^{2} / m \hbar+2 \omega_{\mathrm{G}}\right)$ to the right hand side of (7) is only weakly dependent on the center-ofmass momentum $p_{\mathrm{cm}}$, which permits to replace $p_{\mathrm{cm}}$ by its mean value $p_{\mathrm{cm}}=0$. The remaining integral over the relative momentum $p_{\text {rel }}$ can be evaluated in good approximation by linearizing its argument in the vicinity of $p_{0}$. Setting $p_{\text {rel }}^{2}-p_{0}^{2} \approx 2 p_{0}\left(p_{\text {rel }}-p_{0}\right)$ within the region of non-negligible $\tilde{C}\left(p_{\text {rel }}^{2} / m \hbar+2 \omega_{\mathrm{G}}\right)$ thus yields

$$
\|\tilde{C}\|^{2}=2 \pi T^{2} \Delta p^{2} / p_{0} .
$$

With this, and realizing that the momentum dependent phase factor on the right hand side of (7) effects a free time evolution, we end up with a longitudinal asymptotic wave packet of the form

$$
\left|\Psi_{z}\right\rangle=\left(\widehat{\mathrm{U}}_{z, \tau}^{(0)}+\mathrm{e}^{\mathrm{i} \phi_{\tau}}\right)\left|\Psi_{0}\right\rangle / \sqrt{2}
$$

Clearly, it is the superposition of an early and a late dissociation contribution. Note that the additional free time evolution of the early state component effects a dispersion-induced distortion with respect to the late state component. The inferred single-pulse momentum spectrum reads

$$
\begin{aligned}
\left\langle p_{\mathrm{cm}}, p_{\text {rel }} \mid \Psi_{0}\right\rangle= & \frac{\sqrt{p_{0}} \bar{p}^{2} \operatorname{sinc}\left[\left(p_{\mathrm{cm}}^{2} / 4+p_{\text {rel }}^{2}-p_{0}^{2}\right) / \Delta p^{2}\right]}{\sqrt{\pi} \Delta p\left(p_{\mathrm{cm}}^{2} / 4+p_{\mathrm{rel}}^{2}-p_{0}^{2}+\bar{p}^{2}\right)} \\
& \times\left\langle p_{\mathrm{cm}} \mid \psi_{\mathrm{T}}\right\rangle .
\end{aligned}
$$

The state $\left|\Psi_{0}\right\rangle$ describes a pair of symmetrically counterpropagating particles, in the sense that each particle can propagate in both directions, provided the other particle takes the opposite direction. The corresponding momentum distribution $\left|\left\langle p_{\mathrm{cm}}, p_{\text {rel }} \mid \Psi_{0}\right\rangle\right|^{2}$ is plotted in Fig. 2 for our choice of parameters. As anticipated, the momentum spectrum (10) is sharply peaked at $\left(p_{\mathrm{cm}}, p_{\text {rel }}\right)=\left(0, \pm p_{0}\right)$. This is of great importance for the viability of the DTE Bell test with material particles, as was shown in [19]. 

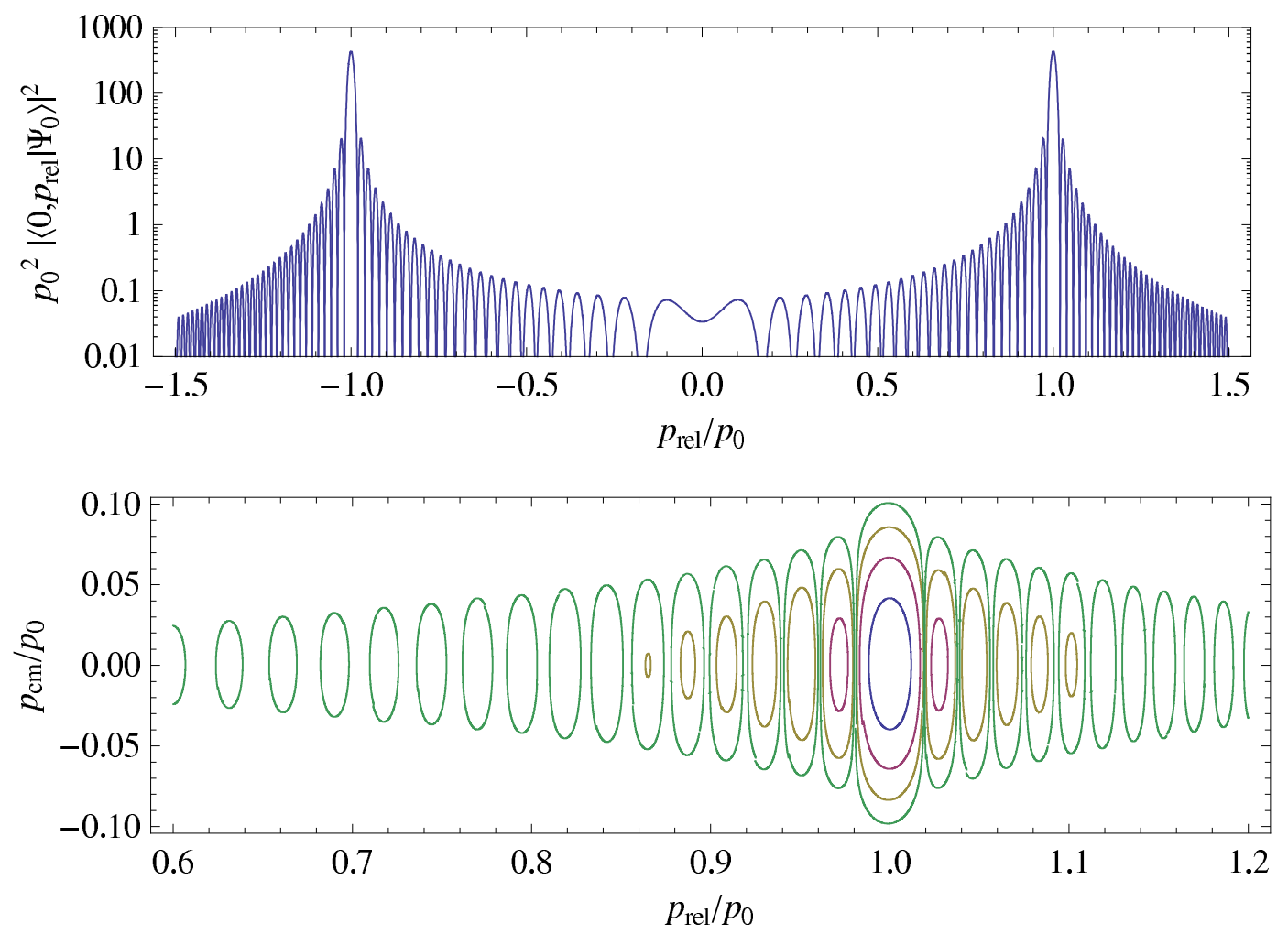

FIG. 2: (color online) Momentum distribution $\left|\left\langle p_{\mathrm{cm}}, p_{\text {rel }} \mid \Psi_{0}\right\rangle\right|^{2}$ for a single Feshbach dissociation pulse with pulse duration $T=60 \mathrm{~ms}$, magnetic field base value $B_{\text {res }}-B_{0}=200 \mathrm{mG}$ and pulse height $\Delta B=400 \mathrm{mG}$. Assuming a longitudinal trap depth of $U_{\mathrm{T}} / k_{B}=50 \mathrm{nK}$ and a transverse trapping frequency of $\omega_{\mathrm{G}} / 2 \pi=300 \mathrm{~Hz}$ this yields the dissociation velocity $p_{0} / \mathrm{m}=5 \mathrm{~mm} / \mathrm{s}$. The upper plot shows the momentum distribution in the $p_{\mathrm{cm}}=0$ plane on a logarithmic scale. As required, the distribution exhibits sharp peaks at $\left(p_{\mathrm{cm}}, p_{\mathrm{rel}}\right)=\left(0, \pm p_{0}\right)$, corresponding to symmetrically counterpropagating particles. The lower logarithmic contour plot focuses on the vicinity of the $\left(p_{\mathrm{cm}}, p_{\mathrm{rel}}\right)=\left(0,+p_{0}\right)$ peak, including the dependence on the center of mass momentum $p_{\mathrm{cm}}$. The width in $p_{\text {rel }}$ is characterized by $\Delta p^{2}=2 m \hbar / T$, with $\left(\Delta p / p_{0}\right)^{2}=0.012$, whereas the width in $p_{\mathrm{cm}}$ is essentially determined by the momentum uncertainty $\sigma_{p, \mathrm{~T}}$ of the longitudinal trap laser ground state $\left|\psi_{\mathrm{T}}\right\rangle$, which follows from the trap frequency $\omega_{\mathrm{T}} / 2 \pi=0.25 \mathrm{~Hz}$, yielding $\sigma_{p, \mathrm{~T}} / p_{0}=0.024$.

The double dissociation pulse sequence generates a two-particle state of the form (9), where due to the additional free time evolution of the early state component the corresponding wave packets trail each other. The early and late wave packets are sufficiently spatially separated such that they can be subjected to the required interferometric transformation. The double pulse dissociation state (9) thus has the structure of a DTE state as described in Section [I. The dissociation probability for the double pulse sequence follows from Eqs. (3) and (8). For our parameters, we get $\left|C_{\mathrm{bg}}\right|^{2} \approx 0.04$, which confirms the premised small dissociation fraction and thus justifies a posteriori the weak coupling approximation in the derivation of (4).

\section{DTE BELL TEST}

In this section we demonstrate explicitly that the DTE state (9), when subjected to the interferometric transfor- mation described in Section I produces an experimentally resolvable fringe pattern that violates a Bell inequality. In brief, the basic idea behind the interferometers is to endow the early state components with an additional phase before recombining them with the late state components. The feasibility of this procedure requires the early and the late wave packets not to overlap before arriving at the first, switchable mirror. If this condition is met by the DTE state (9) the joint probability for detecting a pair of counterpropagating atoms in a particular output port combination labeled by $\sigma_{1}, \sigma_{2}\left(\sigma_{i}= \pm 1\right)$ follows from 


$$
\begin{aligned}
P_{\mathrm{dte}}\left(\sigma_{1}, \sigma_{2} \mid \ell_{1}, \ell_{2}\right)= & \frac{1}{4}\left[1+\sigma_{1} \sigma_{2} \operatorname{Re}\left\{\mathrm{e}^{-\mathrm{i} \phi_{\tau}} \int_{-\infty}^{\infty} \mathrm{d} p_{\mathrm{cm}} \int_{-\infty}^{\infty} \mathrm{d} p_{\text {rel }}\left|\left\langle p_{\text {cm }}, p_{\text {rel }} \mid \Psi_{0}^{(+)}\right\rangle\right|^{2}\right.\right. \\
& \left.\left.\times \exp \left(\mathrm{i} \frac{p_{\text {cm }}\left(\ell_{1}+\ell_{2}\right)}{2 \hbar}+\mathrm{i} \frac{p_{\text {rel }}\left(\ell_{1}-\ell_{2}\right)}{\hbar}-\mathrm{i} \frac{\tau\left(p_{\mathrm{cm}}^{2} / 4+p_{\text {rel }}^{2}\right)}{m \hbar}\right)\right\}\right],
\end{aligned}
$$

as shown in [19]. The two-particle momentum distribution $\left|\left\langle p_{\mathrm{cm}}, p_{\text {rel }} \mid \Psi_{0}^{(+)}\right\rangle\right|^{2}$ derives from the symmetric distribution $\left|\left\langle p_{\mathrm{cm}}, p_{\text {rel }} \mid \Psi_{0}\right\rangle\right|^{2}$ by restricting each particle to propagate into a particular direction, say, particle 1 into positive direction and particle 2 into negative direction. Hence we have

$$
\left|\left\langle p_{\text {cm }}, p_{\text {rel }} \mid \Psi_{0}^{(+)}\right\rangle\right|^{2}=2 \theta\left(p_{\text {rel }}\right)\left|\left\langle p_{\text {cm }}, p_{\text {rel }} \mid \Psi_{0}\right\rangle\right|^{2},
$$

where the additional factor 2 is imposed by normalization. If the two-particle momentum distribution $\left|\left\langle p_{\mathrm{cm}}, p_{\text {rel }} \mid \Psi_{0}\right\rangle\right|^{2}$ is sufficiently well-behaved and the path length differences $\ell_{1}, \ell_{2}$ are chosen such that the early and late wave packets overlap in the output ports, the detection probability (11) reveals a fringe pattern under variation of $\ell_{1}$ and $\ell_{2}$. It is remarkable that the quality of this fringe pattern is only affected by the free time evolution between the early and the late dissociation, whereas any subsequent time evolution does not have any effect, despite of the ongoing broadening of the wave packets.

For the single pulse momentum spectrum (10) of the DTE state (9) the joint detection probability (11) can be evaluated numerically. The resulting fringe pattern for the output port choice $\sigma_{1}=+1$ and $\sigma_{2}=+1$ under variation of $\ell_{1}$ can be seen in Fig. 33.

Specifically, we choose $\ell_{1}=\ell_{0}+\Delta \ell$ and $\ell_{2}=-\ell_{0}$, where $\ell_{0}=\tau p_{0} / m$ defines the optimum overlap path length difference cancelling the separation between the early and the late wave packets. The variation of $\ell_{2}$ or combinations of $\ell_{1}$ and $\ell_{2}$ would of course yield similar fringe patterns. The (constant) relative phase $\phi_{\tau}$ is neglected since it only results in a phase shift of the fringe pattern, while the upper and lower envelope remain unaffected.

Inspecting Fig. 3, we can identify several generic features of the DTE fringe pattern. First, the characteristic length for its period is set by the de Broglie wave length of the relative motion $\lambda_{\text {rel }}=h / p_{0}$. This is natural, since we implement the relative phase shift by displacing the early and late wave packets with respect to each other, while the phase variation of a propagating wave packet is set by its de Broglie wave length. In our case, we get $\lambda_{\text {rel }}=12.4 \mu \mathrm{m}$. This means that, in order to be able to detect the fringe pattern, the interferometers have to be kept stable by about $1 \mu \mathrm{m}$.

The envelope size of the fringe pattern, on the other hand, can be traced back to the unavoidable offset from the optimum wave packet overlap when varying the path length differences. One expects no interference if there
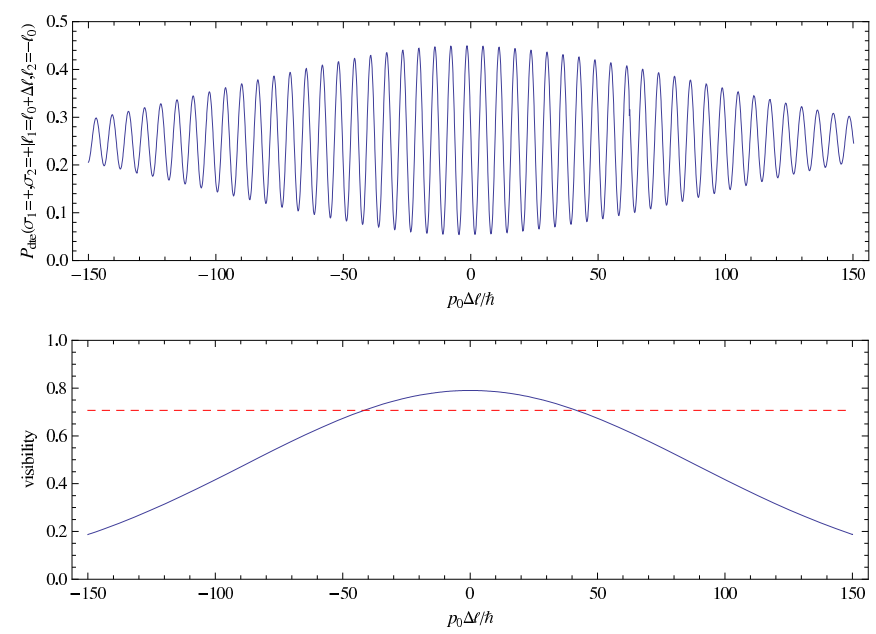

FIG. 3: (color online) Joint probability $P_{\mathrm{dte}}\left(\sigma_{1}, \sigma_{2} \mid \ell_{1}, \ell_{2}\right)$ for detecting a pair of counter-propagating atoms in the output port combination $\sigma_{1}=+1$ and $\sigma_{2}=+1$ under variation of the path length difference $\ell_{1}$. At the outset, $\ell_{1}$ and $\ell_{2}$ are chosen such that optimum overlap of the early and the late wave packets is achieved in the output ports of the beam splitters. The upper plot shows the resulting fringe pattern as it would be seen by the experimenter. The characteristic length for its period is set by the de Broglie wave length of the relative motion, $\lambda_{\text {rel }}=12.4 \mu \mathrm{m}$. The finite range of the envelopes reflects the decreasing wave packet overlap with increasing offset $\Delta \ell$ from the optimum overlap value. The lower plot highlights the corresponding fringe visibility. The dashed line marks the visibility threshold that has to be exceeded in order to violate a Bell inequality. A sufficient number of fringes exceeds the threshold to manifest the corresponding violation.

is no overlap in the beam splitter output ports, resulting in the uncorrelated probability distribution of $25 \%$ per output port combination. Indeed, the width of the fringe pattern, which is of about $200 \mu \mathrm{m}$, matches approximately the initial position spread of the single-particle wave packets. Minor modifications to this can be traced back to the dispersion-induced distortion between the early and the late wave packets.

However, the overall visibility reduction, which prevents the fringe pattern to vary with its maximum amplitude of 0.25 even in the case of optimum overlap, is a genuine effect of the dispersion-induced distortion between the early and the late wave packets. If the early and late wave packets were identical up to displacement, the visibility should not be affected, independently of their shape [22]. It is mainly this criterion which decides whether a 
single pulse momentum distribution is sufficiently wellbehaved or not. Indeed, the joint detection probability (11) can be evaluated analytically in the case of a Gaussian momentum distribution, revealing that the overall visibility is sensitive to the center-of-mass and relative motion momentum spreads $\sigma_{p, \mathrm{~cm}}$ and $\sigma_{p, \text { rel }}$ [19]. Only if these are sufficiently small with respect to the relative momentum $p_{0}$, the resulting fringe pattern can exceed the visibility threshold $1 / \sqrt{2}$ as required for violating a Bell inequality. In our case, the momentum spreads are characterized by $\sigma_{p, \mathrm{~T}} / p_{0}=0.024$ and $\left(\Delta p / p_{0}\right)^{2}=0.012$, yielding a momentum distribution that is sufficiently well peaked to be capable of violating a Bell inequality, as can be seen in the lower plot in Fig. 3. We emphasize that the potential violation sustains over a sufficient number of fringes to be resolvable in experiment.

Another viable approach to attain the expected fringe pattern as determined by a given (sufficiently wellbehaved) momentum distribution is to fit it with a Gaussian. This permits to apply above mentioned analytic result, as was done in [19]. However, for spectra of the form (10) such a least square fit has the tendency to slightly overestimate the accessible visibility. A more conservative procedure than least square fitting would define the fit as an upper envelope of the corresponding momentum distribution. We note that the second momenta of the momentum distribution resulting from (10) are not useful for characterizing the widths of the peaks, while the Gaussian fitting procedure yields a comparatively good approximation in our context.

A final remark should be made on the relative phase $\phi_{\tau}$ between the early and late state component. As can be seen in (11), it also enters the joint detection probability and hence influences the fringe pattern. It was neglected in our numerical investigation since it only effects a phase shift of the fringe pattern. Of course, this assumes that $\phi_{\tau}$ remains constant from shot to shot, while an uncontrolled variation of $\phi_{\tau}$ already on the order of $100 \mathrm{mrad}$ would spoil the fringe pattern. In our Feshbach calculation $\phi_{\tau}$ is determined by

$$
\phi_{\tau} \simeq\left[2 U_{\mathrm{T}} \tau-\mu_{\mathrm{res}} \Delta B T+\mu_{\mathrm{res}}\left(B_{\mathrm{res}}-B_{0}\right) \tau\right] / \hbar+\omega_{\mathrm{G}} \tau .
$$

Hence, we have to require that the magnetic field pulses can be kept stable and are reproducible from shot to shot with a relative accuracy of $10^{-5}$.

\section{CONCLUSIONS}

We described an experimental scenario for the generation of a pair of dissociation-time entangled atoms and the subsequent interferometric Bell test. Assuming viable experimental conditions, the generated state of two counterpropagating atoms was shown to be capable of violating a Bell inequality.

The experiment is based on the Feshbach-induced dissociation of a diatomic molecule, where the entanglement of the dissociating atoms is achieved by applying a sequence of two magnetic field pulses. Hence, no complicated manipulation of the molecular state is required, the only experimental demand is to keep the molecules trapped in a controlled way and to carry out the magnetic field pulses with sufficient accuracy. The atom guides and the required switched, asymmetric Mach-Zehnder interferometers are implemented by laser beams. Since only single-particle interference has to be performed, the interferometers on the opposite sides do not have to be kept stable with respect to each other. This allows to reach macroscopic distances between the two measurement devices, in our scenario on the order of 10 centimeters. The procedure is completed by position measurements that only have to be able to distinguish the chosen beam splitter output port for each atom. This does not require prominent spatial and temporal resolution and is conveniently accomplished by resonant laser beams.

The violation of a Bell inequality on the basis of dissociation-time entanglement would be the manifestation of non-classicality in the motional state of two material particles, without resorting to internal degrees of freedom like spin. Here not only the two atoms are separated on a scale of several centimeters, but also the early and the late wave packets are separated by a distance of $5 \mathrm{~mm}$ on each side. Such a superposition of two consecutively propagating wave packets for each atom, which would be established in this experiment, constitutes a macroscopic violation of the classical particle concept of localization and therefore presents a worthwhile feature of its own.
[1] J. Sherson et al., Nature 443, 557 (2006).

[2] S. Gleyzes et al., Nature 446, 297 (2007).

[3] M. Halder et al., Nature Physics 3, 692 (2007).

[4] D. Moehring et al., Nature 449, 68 (2007).

[5] R. Ursin et al., Nature Physics 3, 481 (2007).

[6] M. Aspelmeyer and K. Schwab, New J. Phys. 10, 095001 (2008).

[7] E. Schrödinger, Proc. Camb. Phil. Soc. 31, 555 (1935).

[8] C. Monroe, D. Meekhof, B. King, and D. Wineland, Science 272, 1131 (1996).
[9] J. Raimond, M. Brune, and S. Haroche, Reviews of Modern Physics 73, 565 (2001).

[10] S. Gerlich et al., 3, 711 (2007).

[11] E. Donley, N. Claussen, S. Thompson, and C. Wieman, Nature 417, 529 (2002).

[12] S. Jochim et al., Science 302, 2101 (2003).

[13] T. Mukaiyama, J. Abo-Shaeer, K. Xu, J. Chin, and W. Ketterle, Phys. Rev. Lett. 92, 180402 (2004).

[14] S. Dürr, T. Volz, and G. Rempe, Phys. Rev. A 70, 031601 (2004). 
[15] T. Köhler, K. Góral, and P. Julienne, Rev. Mod. Phys. 78, 1311 (2006).

[16] K. Jones, E. Tiesinga, P. Lett, and P. Julienne, Rev. Mod. Phys. 78, 483 (2006).

[17] A. Einstein, B. Podolsky, and N. Rosen, Phys. Rev. 47, 777 (1935).

[18] K. V. Kheruntsyan, M. K. Olsen, and P. D. Drummond, Phys. Rev. Lett. 95, 150405 (2005).

[19] C. Gneiting and K. Hornberger, Phys. Rev. Lett 101, 260503 (2008).

[20] J. Brendel, N. Gisin, W. Tittel, and H. Zbinden, Phys.
Rev. Lett. 82, 2594 (1999).

[21] W. Tittel, J. Brendel, H. Zbinden, and N. Gisin, Phys. Rev. Lett. 84, 4737 (2000).

[22] C. Gneiting and K. Hornberger, Appl. Phys. B 95, 237 (2009).

[23] T. Volz et al., Nature Physics 2, 692 (2006).

[24] C. S. Adams, M. Siegel, and J. Mlynek, Phys. Rep. 240, 143 (1994).

[25] H. Kreutzmann et al., Phys. Rev. Lett. 92, 163201 (2004). 